\title{
Experiencia con OK-432 en malformaciones vasculares linfáticas en un hospital del norte de México
}

\author{
Jorge Alberto Cantú Reyes ${ }^{1,2}$, Erik Antonio Mier Escurra ${ }^{1,2 *}$, Joel Cázares Rangel1,2, Julieta Rodríguez De Ita², \\ Jan Lammel Lindemann ${ }^{2,3}$, Luis Francisco Rendón García ${ }^{2}$ y Boris José Chacón López ${ }^{2}$ \\ ${ }^{1}$ Hospital Regional de Alta Especialidad Materno Infantil; ${ }^{2}$ Escuela de Medicina, Tecnológico de Monterrey, Monterrey; ${ }^{3}$ Escuela de Ingeniería, \\ Tecnológico de Monterrey. Nuevo León, México
}

\section{Resumen}

Introducción: Las malformaciones vasculares linfáticas (MVL), anteriormente llamadas linfangiomas, son malformaciones congénitas que se presentan en uno de cada 6,000 a 16,000 nacimientos. El sistema de clasificación más útil para el manejo del linfangioma se basa en el tamaño de los quistes. La resolución espontánea es infrecuente, por lo que el tratamiento expectante no se recomienda. El tratamiento clásico es la cirugía de escisión, pero puede afectar a estructuras vecinas o haber recidivas, por lo que se empezaron a estudiar sustancias esclerosantes, como el OK-432. La mayoría de los estudios incluyen pocos pacientes; los más grandes realizados en México se enfocan a lesiones específicas (macroquísticas) 0 únicamente a una región anatómica. Hasta la fecha, no existen estudios del uso de este fármaco en la población del norte de México. Método: Se describe la experiencia con OK-432 mediante un estudio retrospectivo, descriptivo, en los pacientes con MVL, de 2011 a 2016, en un hospital de referencia del norte de México. Resultados: Veintiséis pacientes con MVL recibieron tratamiento con OK-432. La mayoría fueron macroquísticos (69\%), microquísticos (19\%) y mixtos (12\%). Del total de pacientes, 11 presentaron curación total. El $72 \%$ de la población estudiada tuvo una reducción de > 50\% del tamaño de las lesiones con solo dos aplicaciones de tratamiento; no se presentaron recidivas. Se reportaron complicaciones en dos pacientes (hiperpigmentación de la piel). Conclusiones: El manejo con OK-432 demostró ser efectivo para el tratamiento de las MVL en un hospital de referencia del norte de México.

Palabras clave: OK-432. Linfangioma. Escleroterapia.

\section{Experience with OK-432 in lymphatic vascular malformations in a hospital from northern Mexico}

\begin{abstract}
Background: Lymphatic vascular malformations (LVM) or formerly called lymphangiomas are congenital malformations present in about 1 out of 6000 to -16000 births. The most relevant classification system for lymphangioma management is based on the size of the cysts. Spontaneous resolution is uncommon; thus, expectant management is not recommended. The classic treatment is excisional surgery, but it can affect adjacent structures or have relapses, so, sclerosing substances like OK432 are being studied. The majority of the studies are small in number of patients and are from Japan; the largest studies in
\end{abstract}

Correspondencia:

${ }^{\star}$ Erik Antonio Mier Escurra

E-mail: erik_mier@hotmail.com
Disponible en internet: 13-04-2018 Bol Med Hosp Infant Mex. 2018;75:89-93 www.bmhim.com

1665-1146/C 2018. Hospital Infantil de México Federico Gómez, impreso por Permanyer México SA de CV, todos los derechos reservados. 
Mexico are focused on specific lesions (macrocystic) or a determined anatomical region. To date, there are no studies of the population of the north of Mexico. Methods: The experience with OK-432 was described through a retrospective, descriptive study in patients with LVM, from 2011 to 2016, in a reference hospital of northern Mexico. Results: A total of 26 patients with LVM were treated with OK-432. The majority of the lesions were macrocystic (69\%), microcystic (19\%) and mixed (12\%). From the total number of patients, 11 fully healed, and $72 \%$ of the study population had $>50 \%$ reduction in lesion size, with only 2 applications. There were no recurrences. Complications were reported in 2 patients who had skin hyperpigmentation. Conclusions: OK-432 probed to be an effective treatment for LVM in a reference hospital in the north of Mexico.

Key words: OK-432. Lymphangioma. Sclerotherapy.

\section{Introducción}

Las malformaciones vasculares linfáticas (MVL), anteriormente llamadas linfangiomas, son malformaciones congénitas que se observan en uno de cada 6,000 a 16,000 nacimientos. El $60 \%$ de estas malformaciones pueden verse en la exploración física al nacimiento, y el $80-90 \%$ se detectan cuando el paciente alcanza los 2 años de edad ${ }^{1}$. Estas malformaciones se componen de canales linfáticos y espacios quísticos de tamaño variable. La región de la cabeza y el cuello es el sitio con más frecuencia afectado, pero pueden presentarse en todo el cuerpo. Aunque histológicamente son benignas, estas lesiones pueden extenderse a los órganos circundantes y causar complicaciones potencialmente mortales ${ }^{2}$.

La Sociedad Internacional de Estudio de Anomalías Vasculares clasifica las malformaciones linfáticas como macroquísticas, microquísticas y mixtas; sin embargo, no hay un consenso uniforme al respecto'.

El sistema de clasificación más útil para el manejo de los linfangiomas se basa en el tamaño de los quistes: macroquísticos (quistes $>2 \mathrm{~cm}$ ), microquísticos (quistes $<2 \mathrm{~cm}$ ) o mixtos (cuando más del $50 \%$ del componente total de la enfermedad es macroquístico) ${ }^{3}$. Esta clasificación tiene implicaciones clínicas, ya que los linfangiomas microquísticos parecen ser menos sensibles a los tratamientos clínicos, como la escleroterapia ${ }^{4}$.

El diagnóstico de los linfangiomas es sencillo en la mayoría de los casos. Los tumores se caracterizan por la presencia de una masa blanda, ahulada, compresible, tabicada y mal definida. Los estudios de imagen, ultrasonido, tomografía computarizada o resonancia magnética pueden ayudar al diagnóstico o pueden utilizarse para definir la relación de la lesión con las estructuras vecinas y guiar durante el acto quirúrgico o la aplicación de medicamentos 5 .

Estas malformaciones linfáticas suelen presentar una resolución espontánea de forma muy poco frecuente (hasta el $6 \%$ de los casos) ${ }^{6}$, por lo que el tratamiento expectante no es recomendable ${ }^{1}$. El tratamiento clásico de primera elección es la cirugía de escisión, aunque entre sus inconvenientes se incluyen el daño real 0 potencial estético, o en el peor de los casos el compromiso vascular o nervioso asociado con la lesión, lo que conlleva una escisión parcial y recidivas hasta en el $50 \%$ de los $\operatorname{casos}^{7,8}$. Esto ha propiciado la utilización de otros productos, como el sirolimus, medicamento utilizado como inmunosupresor que se encuentra todavía en estudio para el tratamiento de las $\mathrm{MVL}^{9}$, o los agentes de terapia esclerosante, entre ellos el morruato de sodio, la dextrosa, la tetraciclina, la doxiciclina, la bleomicina, la solución alcohólica de zein (Ethibloc ${ }^{\circledR}$ ) y el OK-432. De acuerdo con Okazaki, et al. ${ }^{2}$, Ogita fue el primero en publicar el uso de OK-432 en los linfangiomas, el cual es derivado de una cepa de Streptococcus pyogenes de baja virulencia tratada con penicilina $G$ potásica. La principal ventaja de OK-432 sobre otros esclerosantes es la ausencia de fibrosis perilesional, por lo que durante los últimos años se ha propuesto como primera línea de tratamiento para las $\mathrm{MVL}^{2}$.

Existe poca literatura mundial en el manejo de MVL con OK-432 debido a su baja frecuencia y a que se presentan solo en pacientes pediátricos, además de que no existe un modelo experimental en animales. Por esto, la mayoría de los estudios se han hecho con pocos pacientes, y los más importantes se han realizado en Japón. Los estudios más grandes en México son de lesiones específicas, como la de tipo macroquístico, o enfocados solo a alguna región anatómica, como la lengua. Con respecto a la población del norte de México, a la fecha no existen estudios publicados. En este estudio se describe la experiencia con OK-432 en los pacientes con $M V L$ en un hospital de referencia del norte de México.

\section{Método}

En un hospital de referencia de la zona norte del país se realizó una revisión de los expedientes de la clínica de MVL. Se analizaron pacientes pediátricos diagnosticados con linfangioma, quienes fueron tratados con OK-432 en un periodo de 6 años (2011 a 2016). Los 
pacientes que se consideraron no candidatos a OK-432 fueron los alérgicos a la penicilina. Se encontraron 35 casos de MVL con diagnóstico clínico comprobado mediante estudios radiológicos. De estos pacientes, se eliminaron nueve debido a que no acudieron a la aplicación del tratamiento, no se presentaron a consultas de seguimiento o tuvieron curación espontanea $(n=3)$. Se analizaron 26 pacientes, quienes fueron tratados con una o más aplicaciones de OK-432 y se les dio seguimiento en el hospital.

El OK-432 se aplicó en las MVL de acuerdo con el protocolo establecido por Ogita, et al. ${ }^{8}$ : se utilizó la presentación de OK-432 de 1 KE y se disolvió con agua inyectable para mantener una densidad de 0.1 $\mathrm{KE} / \mathrm{ml}$. Se anestesió al paciente y se desinfectó el área afectada. Según la accesibilidad de la MVL, se utilizó ultrasonido de apoyo para su aplicación. Se extrajo con una jeringa la mayor cantidad de fluido quístico y se inyectó la misma cantidad en mililitros de la solución OK-432 preparada. La cantidad máxima de medicamento utilizado en la aplicación fue de $20 \mathrm{ml}$.

Después de la aplicación de OK-432, los pacientes permanecieron internados por 24 horas, como mínimo, para el manejo de los posibles eventos adversos, como fiebre, respuesta inflamatoria sistémica y anafilaxia. Se citó a los pacientes 3 semanas después para su valoración; en caso de requerir nueva dosis, se reprogramó para nueva aplicación en 3 semanas. Desde el momento de su curación, se dio al paciente un seguimiento de 5 años.

El análisis de los datos se realizó mediante un vaciado en la base de datos MS Excel ${ }^{\circledR}$ y un graficado con el mismo programa. Los registros de los pacientes fueron revisados con respecto a ubicación, tipo de lesión, presencia de complicaciones o secuelas, número de infiltraciones, estudios de imagen de diagnóstico y extensión.

Los linfangiomas se clasificaron por medio de estudios de imagen en macroquísticos, microquísticos o mixtos. La respuesta se clasificó como completa cuando se logró la total curación de la MVL; favorable, cuando presentó una reducción de más del $50 \%$ del tamaño; desfavorable, cuando presentó una reducción de menos del $50 \%$; y sin respuesta, cuando no hubo disminución de la MVL. Se evaluó la respuesta de la MVL con OK-432 de manera clínica y radiológica.

\section{Resultados}

Los pacientes con MVL tratados con OK-432 que llevaron seguimiento fueron 26 , y de ellos el $62 \%$ de sexo masculino. Las MVL se encontraron anatómicamente en el cuello $(54 \%)$, el tórax $(15 \%)$, las piernas

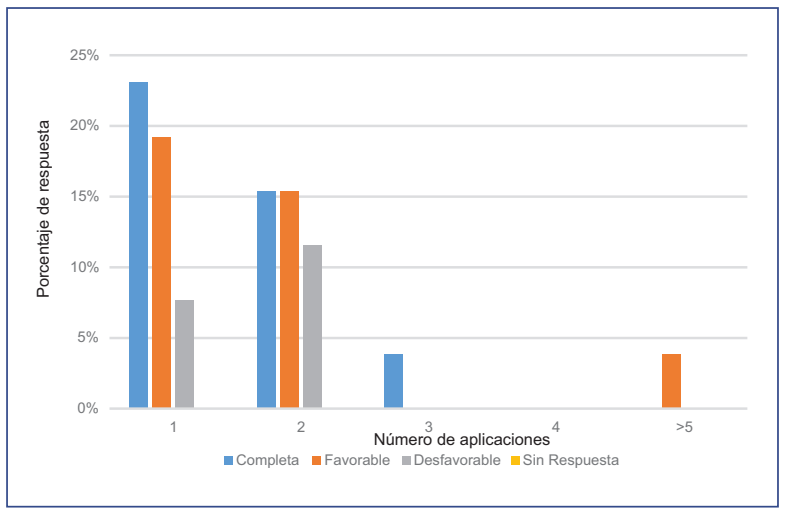

Figura 1. Respuesta según el número de aplicaciones de OK-432 y porcentaje de la población en estudio.

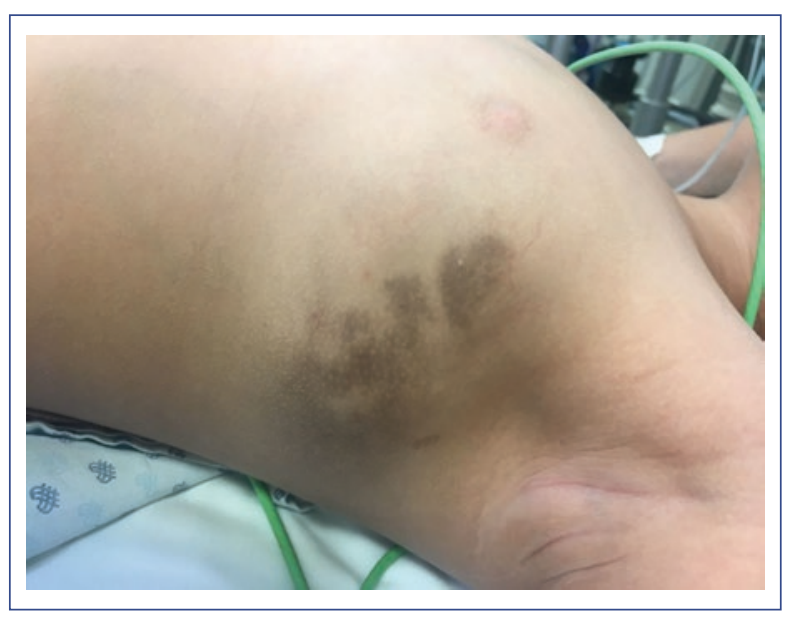

Figura 2. Complicación del tratamiento con OK-432: dermatosis caracterizada por mancha mal definida hiperpigmentada.

(15\%), la cabeza (8\%), el abdomen (4\%) y los brazos (4\%). La distribución por tipo de lesión fue macroquística en el $69 \%$, microquística en el $19 \%$, y mixta en el $12 \%$. El tamaño inicial de las MVL fue, en promedio, de $6 \mathrm{~cm}$ de largo (con un mínimo de $1 \mathrm{~cm}$ y un máximo de $16 \mathrm{~cm}$ ) y $4.6 \mathrm{~cm}$ de ancho (con un mínimo de $0.55 \mathrm{~cm}$ y un máximo de $14 \mathrm{~cm}$ ). El promedio del número de aplicaciones de OK-432 fue de 1.6 (mínimo de una y máximo de cinco aplicaciones) (Fig. 1). Las complicaciones que se presentaron después del tratamiento con OK-432 se manifestaron como una hiperpigmentación de la piel en dos pacientes (Fig. 2).

De los 26 pacientes estudiados, el $72 \%$ presentó una respuesta completa o favorable con solo una o dos aplicaciones; el $4 \%$ presentó respuesta completa a la 
Tabla 1. Respuesta de las malformaciones vasculares linfáticas según el tipo

\begin{tabular}{|l|c|c|c|c|}
\hline Tipo de linfangioma vs. respuesta* & Completa & Favorable & Desfavorable & Sin respuesta \\
\hline Macroquístico & $6 / 18(33 \%)$ & $10 / 18(56 \%)$ & $2 / 18(11 \%)$ & $0 / 18(0 \%)$ \\
\hline Microquístico & $3 / 5(60 \%)$ & $0 / 5(0 \%)$ & $2 / 5(40 \%)$ & $0 / 5(0 \%)$ \\
\hline Mixto & $2 / 3(67 \%)$ & $0 / 3(0 \%)$ & $1 / 3(33 \%)$ & $0 / 3(0 \%)$ \\
\hline
\end{tabular}

*Número total por tipo de linfangioma (porcentaje).

Tabla 2. Respuesta de las malformaciones vasculares linfáticas según la localización anatómica

\begin{tabular}{|l|c|c|c|c|}
\hline Localización de linfangioma vs. respuesta* & Completa & Favorable & Desfavorable & Sin respuesta \\
\hline Cabeza & $1 / 2(50 \%)$ & $1 / 2(50 \%)$ & $0 / 2(0 \%)$ & $0 / 2(0 \%)$ \\
\hline Cuello & $8 / 14(57 \%)$ & $1 / 14(7 \%)$ & $5 / 14(36 \%)$ & $0 / 14(0 \%)$ \\
\hline Tórax & $0 / 4(0 \%)$ & $4 / 4(100 \%)$ & $0 / 4(0 \%)$ & $0 / 4(0 \%)$ \\
\hline Abdomen & $0 / 1(0 \%)$ & $1 / 1(100 \%)$ & $0 / 1(0 \%)$ & $0 / 1(0 \%)$ \\
\hline Piernas & $1 / 4(25 \%)$ & $3 / 4(75 \%)$ & $0 / 4(0 \%)$ & $0 / 4(0 \%)$ \\
\hline Brazos & $1 / 1(100 \%)$ & $0 / 1(0 \%)$ & $0 / 1(0 \%)$ & $0 / 1(0 \%)$ \\
\hline
\end{tabular}

*Número total por sitio anatómico (porcentaje).

tercera aplicación y otro 4\% presentó respuesta favorable a la quinta aplicación (Fig. 1).

Todos los pacientes mostraron respuesta al tratamiento. Once pacientes (42.3\%) tuvieron una respuesta completa, con curación total de la lesión. De las MVL macroquísticas, el 33\% presentó curación completa; las microquísticas tuvieron el $60 \%$ de curación, y las mixtas, el $67 \%$. El $38.5 \%$ de los pacientes presentó una respuesta favorable, encabezada por las MVL macroquísticas, y el 19.2\% tuvo una respuesta desfavorable, encabezada por las MVL microquísticas, por lo que las lesiones mostraron mucha variabilidad dependiendo del paciente (Tabla 1).

Según la localización anatómica de las MVL, la del brazo presentó curación completa; sin embargo, esta localización anatómica de MVL solo la tuvo un paciente. Las lesiones en el cuello presentaron un $57 \%$ de curación completa, y las lesiones en la cabeza, un $50 \%$ de curación completa. Se obtuvo el porcentaje de curación de acuerdo con la localización anatómica y el tipo de respuesta al tratamiento (Tabla 2).

\section{Discusión}

En este estudio, que representa una muestra de la demografía del norte del país, se observó un mayor porcentaje de MVL en el sexo masculino $(62 \%)$, lo cual difiere con otros estudios que muestran una proporción similar en ambos sexos ${ }^{1-3,10}$. El cuello y la cabeza fueron los sitios anatómicos más comunes de localización de las MVL, y el tipo de lesión más frecuente fue la de tipo macroquístico. Estos datos, además del tamaño de las lesiones, muestran resultados similares a los otros estudios realizados previamente ${ }^{1,2,10}$. En este estudio, el promedio de aplicaciones del tratamiento con OK-432 fue de 1.6, menor que el número de aplicaciones reportadas en la mayoría de los estudios 2,10 .

Se presentaron complicaciones en dos pacientes, 10 cual se manifestó como una hiperpigmentación de la piel. Comparados con otros estudios publicados, los resultados varían ${ }^{1,2,10}$, ya que algunos consideran como complicaciones el hecho de que el paciente presente fiebre 0 edema, malestar e hipertermia, en el área de la lesión. En este estudio, como en otra literatura existente ${ }^{3}$, se manejaron estos últimos como reacciones secundarias.

De los 26 pacientes a los que se aplicó OK-432, el $72 \%$ presentó una respuesta completa o favorable con solo una o dos aplicaciones. Estos resultados son semejantes a los obtenidos en algunos estudios ${ }^{1,2}$. La mayoría de los trabajos concluyen que, si no hay respuesta con la primera dosis, es cada vez menos probable que esta se logre con dosis subsecuentes ${ }^{1,2}$. Sin embargo, si no se consiguió la respuesta esperada con la primera dosis, 
se puede seguir administrando OK-432 en citas posteriores sin un límite establecido de aplicaciones máximas. En el estudio de Okazaki, et al. ${ }^{2}$, el cual es uno de los más importantes realizados en Japón, se reportan hasta 23 aplicaciones a un mismo paciente. Por esta razón, muchos de los pacientes que aún no presentaron curación completa seguirán con aplicaciones posteriores.

En todos los pacientes a quienes se aplicó OK-432 se observó una disminución del tamaño de la lesión; sin embargo, la calidad de la respuesta obtenida fue muy variable, y no se encontró una diferencia significativa entre los tipos de MVL. En la gran mayoría de los estudios, la lesión macroquística es la que presenta mejor respuesta con OK-432 ${ }^{1-3}$. En un estudio de Mejía, et al. ${ }^{10}$, en el que se trataron específicamente MVL de tipo macroquístico, se describió su alta efectividad en pacientes mexicanos ${ }^{10}$. En algunos estudios, las lesiones microquísticas presentan muy pobre respuesta ${ }^{1-3}$, y en otros no se encontraron diferencias de respuesta dependiendo de la lesión ${ }^{11}$. Sin embargo, en este estudio, el 33\% de las lesiones macroquísticas presentaron curación completa, el $60 \%$ de las microquísticas y el $67 \%$ de las mixtas, aunque la MVL macroquística mostró más respuestas favorables y menos desfavorables que las demás. Además de la variabilidad de las respuestas, existe una gran diferencia entre el número de pacientes con los diferentes tipos de malformación: 18 con macroquística, cinco con microquística y tres con mixta.

Dependiendo de la localización anatómica, los resultados fueron variables en cuanto a la respuesta al tratamiento con OK-432, sin presentarse una diferencia significativa. En otros estudios en los que también realizaron esta comparación, el resultado no fue significativo ${ }^{2}$ o no hubo diferencia en cuanto a la localización de la lesión y el resultado obtenido con el tratamiento con OK-432'.

Una de las limitantes de este estudio es su naturaleza retrospectiva, además de que solo se aplicó tratamiento con OK-432. Por lo mismo, no se puede realizar una comparación directa con otros tratamientos.

Mediante los resultados obtenidos se concluye que, sin importar el tipo, el tamaño ni la localización anatómica, todos los pacientes con MVL presentaron mejoría o curación de la lesión, por lo que el tratamiento con OK-432 mostró ser un método efectivo para las MVL en un hospital de referencia del norte de México.

\section{Responsabilidades éticas}

Protección de personas y animales. Los autores declaran que los procedimientos seguidos se conformaron a las normas éticas del comité de experimentación humana responsable y de acuerdo con la Asociación Médica Mundial y la Declaración de Helsinki.

Confidencialidad de los datos. Los autores declaran que han seguido los protocolos de su centro de trabajo sobre la publicación de datos de pacientes.

Derecho a la privacidad y consentimiento informado. Los autores han obtenido el consentimiento informado de los pacientes y/o sujetos referidos en el artículo. Este documento obra en poder del autor de correspondencia.

\section{Agradecimientos}

Al equipo de médicos y enfermería del Hospital Regional Materno Infantil de Alta Especialidad, por su apoyo y participación en la evaluación de los pacientes.

\section{Financiamiento}

No se cuenta con ninguna fuente de financiamiento.

\section{Conflicto de intereses}

Los autores declaran no tener ningún conflicto de intereses.

\section{Bibliografía}

1. de Oliveira Olímpio H, Bustorff-Silva J, de Oliveira Filho AG, de Araujo KC. Cross-sectional study comparing different therapeutic modalities for cystic lymphangiomas in children. Clinics. 2014;69:505-8.

2. Okazaki T, Iwatani S, Yanai T, Kobayashi H, Kato Y, Marusasa $T$, et al. Treatment of lymphangioma in children: our experience of 128 cases. J Pediatr Surg. 2007;42:386-9.

3. Giguère CM, Bauman NM, Sato Y, Burke DK, Greinwald JH, Pransky S, et al. Treatment of lymphangiomas with OK-432 (Picibanil) sclerotherapy: a prospective multi-institutional trial. Arch Otolaryngol Head Neck Surg. 2002;128:1137-44.

4. Wassef M, Blei F, Adams D, Alomari A, Baselga E, Berenstein A, et al. Vascular anomalies classification: recommendations from the International Society for the Study of Vascular Anomalies. Pediatrics. 2015;136:203-14.

5. Grasso D, Pelizzo G, Zocconi E, Schleef J. Lymphangiomas of the head and neck in children. Acta Otorhinolaryngol Ital. 2008;28:17-20.

6. Rebuffini E, Zuccarino L, Grecchi E, Carinci F, Merulla VE. Picibanil (OK-432) in the treatment of head and neck lymphangiomas in children. Dent Res J (Isfahan). 2012;9:S192-6.

7. Torres-Palomino G, Juárez-Domínguez G, Méndez-Sánchez L. Escleroterapia en malformaciones linfáticas infantiles: revisión sistemática de la literatura. An Med. 2014;59:127-32.

8. Ogita S, Mejía M, Sánchez J. OK-432 Picibanil. Linfangiomas. Disponible en: http://linfangiomas.com/default.htm

9. Lackner $H$, Karastaneva A, Schwinger W, Benesch M, Sovinz $P$, Seidel M, et al. Sirolimus for the treatment of children with various complicated vascular anomalies. Eur J Pediatr. 2015;174:1579-84.

10. Mejía M, Sánchez J, Reyes R, Lezama P, Ogita S, Bracho E, et al. Tratamiento de linfangiomas con OK432 (Picibanil). Arch Inv Pediatr Mex. 2005;8:5-9.

11. Santana C, Ambriz G, Ramírez E, López S, Torres E. Experiencia con el uso de OK-432 para el tratamiento de malformaciones linfáticas en niños. XLVIII Congreso Nacional de Cirugía Pediátrica; 2015. 